\title{
A rare case of unruptured ectopic pregnancy in a rudimentary horn with a dead foetus of sixteen weeks
}

\author{
Rama Saraladevi*, B. Vijaya Laxmi, J. Madhavi, P. Radha
}

Department of Obstetrics and Gynaecology, Kakatiya Medical College, Warangal, Telangana, India

Received: 04 November 2015

Revised: 20 January 2016

Accepted: 23 January 2016

\section{*Correspondence:}

Dr. Rama Saraladevi,

E-mail: saraladevi994@gmail.com

Copyright: ( $)$ the author(s), publisher and licensee Medip Academy. This is an open-access article distributed under the terms of the Creative Commons Attribution Non-Commercial License, which permits unrestricted non-commercial use, distribution, and reproduction in any medium, provided the original work is properly cited.

\begin{abstract}
Ectopic pregnancy in a rudimentary horn of bicornuate uterus is a rare type of ectopic pregnancy. It also called as cornual pregnancy. It is difficult to differentiate from tubal pregnancy. The clinical features are similar to that of tubal ectopic pregnancy. The diagnosis is made by operative finding, that is, position of the attachment of the round ligament to the gestational sac, which is always lateral to the cornual pregnancy, whereas in tubal pregnancy the round ligament lies medial to the gestational sac.
\end{abstract}

Keywords: Ectopic gestation, Rudimentary horn, Misoprostol

\section{INTRODUCTION}

Ectopic in a rudimentary horn is a rare type of ectopic pregnancy. It's incidence is 1 in 3000-6000. ${ }^{1}$ The fate of pregnancy depends upon degree of development of rudimentary horn. If the rudimentary horn is ill developed, the muscle wall becomes thinned \& gets ruputred during pregnancy. These complications occur during $4^{\text {th }}$ month and cause severe haemorrhage. The diagnosis is confusing and difficult to differentiate from tubal pregnancy. The diagnosis is made during surgery by noticing the position of round ligament to gestational sac. $^{2,3}$

Hence rudimentary horn ectopic is a diagnostic \& therapeutic challenge with potential severe consequences if uterine rupture occurs with massive intra-abdominal heamorrhage.

\section{CASE REPORT}

We are reporting this case of rudimentary horn ectopic gestation because of its unusual presentation in second trimester which led to diagnostic dilemmna and mismanagment.

A 25 yrs old $\mathrm{g}_{2} \mathrm{a}_{1}$ woman at 16 weeks of gestation referred to GMH, hanamkonda with h/o 4 months amenorrhea, went to a private doctor for ante-natal check up, where the doctor did ultrasound examination and misdiagnosed as intra-uterine death of 16 weeks gestation. She took consent for $\mathrm{mtp}$ and gave mifepristone $200 \mathrm{mg}$ followed by $\mathrm{pge}_{1}$. There was no vaginal bleeding or expulsion of products of conception or uterine contractions.

Patient was comfortable and haemodynamically stable. Then the private doctor reffered this case to government maternity hospital, hanamkonda. The duty doctor did all the investigations and ultrasound examination again.

She also misdiagnosed as intra uterine death of 16 weeks foetus. She attempted to do MTP by instillation of extraamniotic ethacridine lactate (because of failed mifepristone \& misoprostol). But she was unable to pass the foley's catheter beyond 2" then after filling the 
bladder; careful ultrasound examination was done \& diagnosed as unruptured tubal ectopic pregnancy on left side with 16 weeks gestation dead fetus and normal sized uterus on right side.

Emergency laparotomy was done and both the tubes and ovaries appeared normal. The pregnancy was in the rudimentary horn on left side of normal sized uterus (Figure 1). It was diagnosed by identifying the relation of round ligament to the gestational sac.

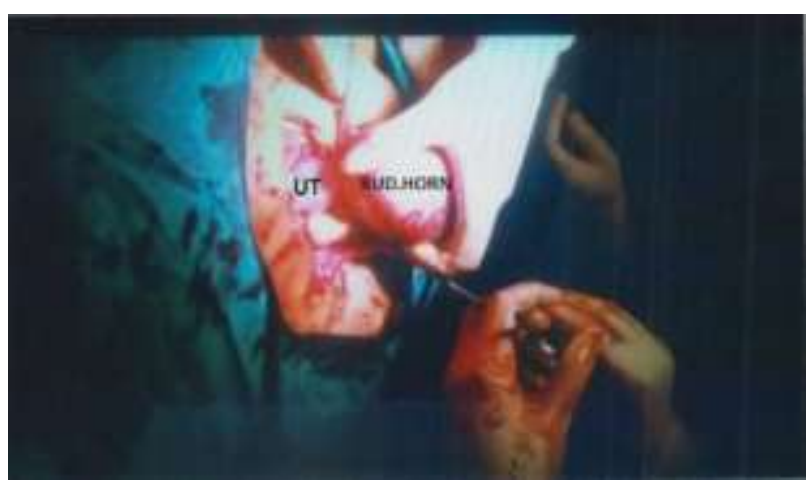

Figure 1: Intra operative picture showing rudimentary horn on left side.

Rudimentary horn was connected to the uterus with a pedicle \& there was no communication with the uterine cavity. The rudimentary horn was resected and sent for histo-pathological examination.

Cut-Section shows thickened musculature around the gestational sac with dead fetus (Figure 2).

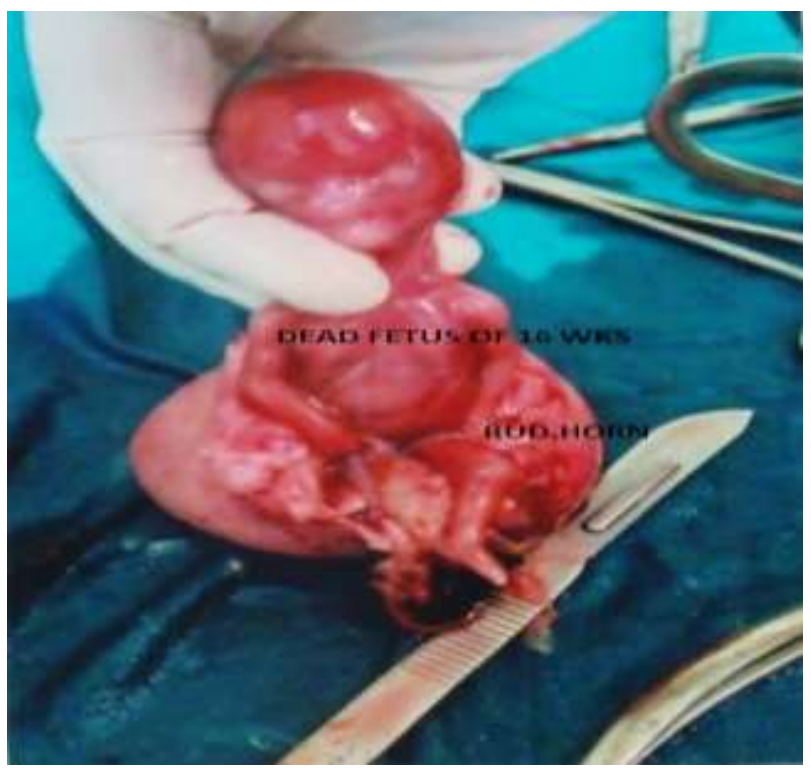

Figure 2: Cut section of rudimentary horn.

H.P.E shows thick musculature surrounding gestational sac, suggestive of uterine musculature.
Post-operative period was uneventful. Patient was discharged on $7^{\text {th }}$ POD. She reviewed at OPD with no complaints.

Pregnancy in rudimentary horn of bicornuate uterus is always confused with tubal pregnancy, but diagnosis is confirmed by finding the relation of round ligaments to gestational sac.

\section{DISCUSSION}

Pregnancy in a rudimentary horn of bicornuate uterus is called corunal pregnancy. ${ }^{4}$ The horn doesn't usually communicate with the uterine cavity the impregnation is presumed to occur by a spermatozoan which passes through the normal $1 / 2$ of the uterus and tube. It is then fertilized by an ovum in the peritoneal cavity or in the tube connected to the rudimentary horn. ${ }^{5}$

The concerned ovum is usually shed from the ovary on the same side of rudimentary horn. The general\& local reactions are similar to those in the tubal pregnancy. But these are intensified and pregnancy will continue for long time. Termination by rupture is inevitable between 12-20 weeks with massive intraperitoneal haemorrhage.

Correct diagnosis of advanced rudimentary horn ectopic warrants high index of suspicion because of symptoms are often non-specific and diagnostic imaging is inconclusive. Ultrasound imaging is most important diagnostic tool. MRI made an additional examination. Even then, its diagnostic accuracy is low. Diagnostic laparoscopy is the ideal diagnostic tool for the diagnosis of ectopic pregnancy. Colour Doppler improves diagnostic sensitivity to $70-80 \%$ for ectopic pregnancy in comparison to ultrasound. $3 \mathrm{~d}$ USG is also an important tool for ectopic. ${ }^{6,7}$

The diagnosis is seldom done before laparotomy. The condition is commonly confused with tubal pregnancy, fibroid, ovarian tumour complicating pregnancy. Even during laparotomy, it is confused with interstitial pregnancy. Position of round ligaments attachments with sac and the long pedicle with which it is attached to the uterus are the diagnostic points.

In our case, since death of the foetus occurred at 16 weeks, rupture is not expected to occur. Otherwise the patient might have landed in catastrophe. In our case, rudimentary horn was connected to the uterus with a pedicle and there is no communication between the uterine cavity and rudimentary horn. This also explains the transperitoneal migration which is one of the etiological factors for ectopic gestation.

\section{Management}

Management includes removal of the rudimentary horn with pregnancy if the pedicle is short. If the attachment is wide, hysterectomy may have to be done. Cornual 
resection and repair of the defect are possible in $>50 \%$ of cases. But when extensive resection of myometrium is required to preserved the uterus the surgical risk is high and the further risk of uterine rupture in subsequent pregnancy should be considered., ${ }^{2,5}$

\section{CONCLUSION}

Advanced unruptured ectopic gestation in rudimentary horn with dead foetus explained in this case is always an emergency and should be treated with laparotomy or laparoscopic surgery. We have done laparotomy and resected the rudimentary horn along with the foetus in situ.

Correct diagnosis of an advanced ectopic pregnancy warrants a high index of suspicion because of symptoms are often non-specific and diagnostic imaging is inconclusive.

This case is a learning lesson to the treating doctors from a mistaken diagnosis of I.U.D (Intra Utrine Death) instead of rudimentary horn ectopic gestation. In early $1^{\text {st }}$ trimester USG along with the confirmation of intrauterine pregnancy we have to role out uterine anomalies or otherwise preconceptional diagnosis of utrine anamalies essential especially in patients with previous h/o abortions by which we can avoid this sort of mismanagement and we can treat the patient appropriately.
Funding: No funding sources Conflict of interest: None declared

Ethical approval: Not required

\section{REFERENCES}

1. Arup Kumar Mahji's bedside clinics in Obstetrics. 2011;7,3(10):3-14.

2. Jeffcoate's Principles of Gynecology. 2014;8:144.

3. Mudaliar, Menon's clinical Obstetrics. 2015;12:153.

4. Text book of obstetrics by D.C Dutta. 2015;8:202.

5. Howkins \& Bourne Shaw's text book Gynecology: 2013;15:270.

6. Kurjak A, Frank FA. Donald School text book of ultrasound on obstetrics Gynecology. 2003;1:176-7.

7. Berek \& Novak's Gynecology. 2013;15:631-6.

8. TeLinde's Operative Gynecology. 2011;10:816-7.

9. Sant CLH Andersen PE. Misdiagnosed uterine ruptured of an advanced Cornual Pregnancy case reporting in Radiology. 2012.

Cite this article as: Saraladevi R, Vijaya Laxmi B, Madhavi J, Radha P. A rare case of unruptured ectopic pregnancy in a rudimentary horn with a dead foetus of sixteen weeks. Int J Reprod Contracept Obstet Gynecol 2016;5:566-8. 